\title{
Fisioterapia na disfunção urinária infantil: revisão sistemática da literatura nacional
}

\author{
Physiotherapy on bladder dysfunction in children: \\ systematic review of Brazilian clinical trials
}

\author{
Mayara Vieira Souza Halate ${ }^{1}$ \\ Erica Feio Carneiro Nunes ${ }^{1}$ \\ Gustavo Fernando Sutter Latorre ${ }^{1}$
}

\section{RESUMO}

Contexto: Disfunções urinárias infantis, definidas como anormalidades na fase de enchimento ou esvaziamento da bexiga, trazem grandes problemas para a qualidade de vida dos pacientes, ocasionando problemas físicos com risco de maior comprometimento renal, e problemas emocionais. Apesar da Fisioterapia Pélvica (FP), no tratamento destas crianças, estar se destacando, especialmente por causa de seus bons resultados, ainda não estão claras quais técnicas são as mais utilizadas e mais eficazes para este fim. Metodologia: trata-se de uma revisão sistemática sobre as técnicas fisioterápicas para o tratamento da disfunção urinária infantil restrita a ensaios clínicos, publicados na língua portuguesa, nas bases-de dados SciELO, LILACS e Data CAPES. Resultados: foram encontrados 59 artigos, dos quais apenas seis tratavam sobre técnicas fisioterápicas, sendo três duplicados entre as bases. Um descrevia eletroestimulação para incontinência urinária (IU) de origem neurogênica, um sobre cinesioterapia para IU funcional e outro sobre uroterapia para IU de ambas as origens, todos apresentando bons resultados. Conclusão: pequeno número de ensaios clínicos impossibilitou comparações, mas todas as técnicas apresentaram bons resultados, inclusive superiores à medicação no tratamento da disfunção em questão. A bibliografia nacional é demasiadamente escassa, sendo fundamental novos ensaios clínicos especialmente sobre a eficácia das técnicas.

\section{PALAVRAS-CHAVE:}

criança. incontinência urinária. fisioterapia

${ }^{1}$ Universidade do Estado do Pará. 


\begin{abstract}
Background: Children urinary dysfunction, defined as abnormalities in the emptying or filling phases of the bladder, brings serious problems due to the quality of life, causing greater risk of renal impairment and emotional problems. Although the pelvic physiotherapy for these children be increasing, especially due to the good results, effectiveness and frequency of the techniques are not clearly defined. Methodology: systematic review of physiotherapy techniques for the treatment of childhood urinary dysfunction in the Scielo, LILACS and DataCAPES databases, restricted to clinical trials in Portuguese. Results: 59 articles, of which only six treated on physiotherapy techniques, three repeated between the bases. One described electrostimulation for UI neurogenic origin, one on cinesioterapia to functional $\mathrm{UI}$ and another on uroterapia for $\mathrm{UI}$ from both sources, every one describing good results. Conclusion: Low number of clinical trials impossible comparisons, but all the techniques presented good results, including superior to medication in the treatment of dysfunction in question. The national bibliography is too scarce, with new pivotal clinical trials especially on the effectiveness of the techniques.
\end{abstract}

\title{
KEYWORDS:
}

Child; Urinary incontinence; Physical Therapy Specialty. 


\section{INTRODUÇÃO}

A perda de urina em crianças é uma situação comumente identificada na prática clínica pediátrica'. No entanto, esse problema somente é levado em consideração quando associado às infecções do trato urinário inferior (ITUI), principalmente as de repetição, pelo fato de representarem grande risco para o trato urinário superior e a saúde em geral². Sobretudo trata-se de um problema de ordem social, que pode gerar isolamento da criança em relação a outras crianças ou aos próprios familiares ${ }^{3}$.

A disfunção do trato urinário inferior (DTUI) indica uma função vesical anormal para a idade da criança, se constituindo como um distúrbio nas fases de enchimento ou esvaziamento vesical, cuja origem pode ser neurogênica, miogênica ou funcional, mas nem sempre sintomática ${ }^{4}$.

São considerados distúrbios de armazenamento aqueles em que contrações vesicais involuntárias aparecem no enchimento vesical. Contudo, a micção é coordenada, o jato urinário é forte e não há resíduo pós miccional. Já os problemas na fase do esvaziamento, particularmente a disfunção miccional, caracterizada por uma micção não coordenada, o jato urinário é fraco e o resíduo pós miccional tende a estar presente, ou a bexiga hipoativa, normalmente relacionada à postergação da micção ${ }^{5}$.

Segundo a Sociedade Internacional de Continência Infantil (ICCS) as crianças até os quatro anos de idade já devem ter adquirido a continência urinária diurna, e até os cinco anos a continência urinária noturna. Por razões desconhecidas, em algumas crianças pode haver o retardo do amadurecimento neurofisiológico das vias da micção ${ }^{3}$.

No Brasil, dois estudos identificaram a prevalência de sintomas miccionais em crianças com faixas etárias distintas, sendo que de 3-9 anos uma prevalência de $22,8 \%$, e destes $10,5 \%$ meninos e $33,8 \%$ de meninas ${ }^{1}$, e entre $6-12$ anos a prevalência foi de $21,8 \%$, sendo, $22,4 \%$ meninos e $77,6 \%$ meninas 5 . Estes dados corroboram com o observado na literatura internacional, em que $20 \%$ a $30 \%$ das crianças apresentam sintomas relacionados à disfunção urináriaa, ${ }^{6,7,8}$.

O tratamento dos pacientes com disfunção urinária pode ser farmacológico e/ou cirúrgico, treina- mento com alarmes, uroterapia, no qual tanto as crianças quanto seus pais são orientados sobre o posicionamento miccional, hábitos urinários, ingestão de líquidos, prevenção de constipação, preenchimento do diário miccional e o treinamento da musculatura do assoalho pélvico (TMAP), auxiliado ou não por biofeedback e eletroestimulação. Estas formas de tratamentos são consideradas seguras e bem aceitáveis para as crianças. Cada modalidade vai ser selecionada de acordo com a falha funcional em questão ${ }^{3}$.

No entanto, apesar do aparente consenso a respeito da necessidade de diferenciação no tratamento da criança e dos bons resultados da terapia conservadora da fisioterapia, ainda não estão claras quais técnicas são as mais utilizadas e mais eficazes para tratar a disfunção urinária infantil. A existência de diversos tipos de técnicas para uma gama relativamente pequena de problemas pode indicar falta de otimização terapêutica.

Ante o exposto o presente estudo se propôs revisar a literatura nacional em busca das técnicas mais utilizadas no Brasil no tratamento das disfunções urinárias infantis, discutindo a eficácia das mesmas com base nos resultados clínicos apresentados.

\section{METODOLOGIA}

Foram pesquisados estudos do tipo ensaio clínico nas bases de dados bases de dados SciELO, LILACS e DataCAPES, utilizando as palavras chaves "incontinência urinária" e "crianças", bem como suas variantes em inglês. Os critérios de inclusão dos estudos foram os seguintes: ensaios clínicos publicados em língua portuguesa que apresentassem a atuação da fisioterapia em qualquer tipo de disfunção urinária em crianças, sendo excluídos artigos duplicados nas bases de dados.

Os artigos resultantes foram organizados de acordo com autor, ano, amostra, objetivos, disfunção abordada, técnicas utilizadas e resultados. A comparação da eficácia das técnicas seria realizada de maneira analítica, de acordo com o tamanho da amostra e o tipo de estatística utilizada, no entanto o baixo número de artigos primários aliado às diferenças metodológicas e, particularmente, quanto ao 
tipo de disfunção tratada, impossibilitou comparações mais acuradas.

\section{RESULTADOS}

A pesquisa inicial totalizou 53 artigos, a maioria deles repetidos entre as bases. Na base Scielo foram encontrados 31 artigos, dos quais 28 não foram incluídos por se tratarem de diagnóstico através de exames de imagem, e de tratamento de IU em mulheres, ou por não se tratarem de ensaios clínicos, restando três artigos incluídos. Na busca realizada na base DataCAPES foram identificados 13 artigos, dos quais dez não foram incluídos por se tratarem principalmente de estudos sobre diagnóstico e doença renal, sendo que três estudos, foram excluídos por estarem repetidos na base SciELO. As buscas na base BVS/LILACS encontraram 19 artigos, dos quais nenhum foi incluído, uma vez que 16 deles tratavam de cirurgia, diagnóstico especialmente em adolescentes, e três por erro da base - artigos não puderam ser abertos. Os resultados estão expostos no quadro 1.

Quadro 1: Artigos em língua portuguesa sobre o tratamento de disfunções urinárias em crianças, de acordo com autor, ano, amostra, tipo de disfunção, técnicas utilizadas e resultados

\begin{tabular}{|c|c|c|c|c|}
\hline $\begin{array}{l}\text { A U T O R / } \\
\text { ANO }\end{array}$ & OBJETIVOS & METODOLOGIA & RESULTADOS & CONCLUSÃO \\
\hline $\begin{array}{l}\text { Kajbaf za- } \\
\text { deh, et al. }{ }^{9}\end{array}$ & $\begin{array}{l}\text { Investigar a eficácia da } \\
\text { eletroestimulação fun- } \\
\text { cional (FES) nos sinto- } \\
\text { mas miccionais em } \\
\text { crianças com mielome- } \\
\text { ningocele que apre- } \\
\text { sentam IU }\end{array}$ & $\begin{array}{l}\text { Seis meninas e seis meninos } \\
\text { foram tratados somente com } \\
\text { FES ( } 40 \mathrm{~Hz} \text { e } 250 \mu \mathrm{s} \text { ) com ele- } \\
\text { trodos transcutâneos autoa- } \\
\text { desivos, sendo o eletrodo po- } \\
\text { sitivo posicionado acima da } \\
\text { sínfise púbica e o negativo } \\
\text { sobre a uretra, por } 3 \text { meses, } 3 \\
\text { sessões semanais. Resultados } \\
\text { medidos por exame urodinâ- } \\
\text { mico }\end{array}$ & $\begin{array}{l}\text { 9/12 crianças apre- } \\
\text { sentaram melhora } \\
\text { no índice de IU. }\end{array}$ & $\begin{array}{l}\text { A FES pode ter efeitos } \\
\text { positivos sobre os } \\
\text { sintomas miccionais, } \\
\text { sendo um método } \\
\text { eficaz, de baixo custo } \\
\text { e menos invasivo, } \\
\text { melhor tolerado pe- } \\
\text { las crianças. }\end{array}$ \\
\hline $\begin{array}{l}\text { Azevedo et } \\
\text { al. }{ }^{10}\end{array}$ & $\begin{array}{l}\text { Avaliar a eficácia das } \\
\text { condutas adotadas pe- } \\
\text { la equipe interdiscipli- } \\
\text { nar para o tratamento } \\
\text { de crianças e adoles- } \\
\text { centes com DTUI. }\end{array}$ & $\begin{array}{l}192 \text { pacientes com DTUI com } \\
\text { média etária } 6,6 \text { anos recebe- } \\
\text { ram medicação e/ou, urotera- } \\
\text { pia (comportamental, biofee- } \\
\text { dback e eletroestimulação sa- } \\
\text { cral) ou cirúrgico na falha dos } \\
\text { conservadores. } 60,4 \% \text { de ori- } \\
\text { gem neurogênica }\end{array}$ & $\begin{array}{l}\text { Uroterapia mostrou } \\
\text { redução significativa } \\
\text { de ITUI, da IU diurna, } \\
\text { da enurese noturna, } \\
\text { da incontinência fe- } \\
\text { cal e do refluxo vesi- } \\
\text { co ureteral. }\end{array}$ & $\begin{array}{l}\text { O tratamento deve } \\
\text { ser individualizado e } \\
\text { entregue em um cen- } \\
\text { tro de atendimento } \\
\text { especializado inter- } \\
\text { disciplinar. }\end{array}$ \\
\hline $\begin{array}{l}\text { Campos et } \\
\text { al. }{ }^{11}\end{array}$ & $\begin{array}{l}\text { Comparar fisioterapia } \\
\text { com anticolinérgicos } \\
\text { para disfunção miccio- } \\
\text { nal de origem funcio- } \\
\text { nal }\end{array}$ & $\begin{array}{l}47 \text { crianças de } 5 \text { a } 10 \text { anos fo- } \\
\text { ram divididas em dois grupos, } \\
21 \text { crianças tratadas com an- } \\
\text { ticolinérgicos, e } 26 \text { crianças } \\
\text { por TMAP uma vez na semana } \\
\text { por fisioterapeuta e duas ve- } \\
\text { zes domiciliar. Ambos recebe- } \\
\text { ram terapia comportamental. }\end{array}$ & $\begin{array}{l}\text { Após } 4 \text { meses do tér- } \\
\text { mino do tratamento } \\
\text { observou-se que } \\
31,83 \% \text { das crianças } \\
\text { tratadas com oxibu- } \\
\text { tinina foram conti- } \\
\text { nentes dia e noite, } \\
\text { contra } 58,33 \% \text { das } \\
\text { crianças que realiza- } \\
\text { ram FP e terapia } \\
\text { comportamental fo- } \\
\text { ram curados. }\end{array}$ & $\begin{array}{l}\text { TMAP mostrou ser } \\
\text { eficaz, não invasivo e } \\
\text { facilmente aceito pe- } \\
\text { las crianças e associa- } \\
\text { da a terapia compor- } \\
\text { tamental e mostrou } \\
\text { melhora da IU supe- } \\
\text { rior ao uso de oxibu- } \\
\text { tinina e terapia com- } \\
\text { portamental da enu- } \\
\text { rese não monossinto- } \\
\text { mática }\end{array}$ \\
\hline
\end{tabular}


Kajbafzadeh et al. ${ }^{9}$ investigaram a eficácia da FES sobre a incontinência urinária severa secundária à mielomeningocele em doze crianças, seis de cada gênero. O sucesso do tratamento, que consistiu em três sessões semanais durante três meses, foi medido por meio de ultrassonografia e eletromiografia de superfície, sendo observados especialmente a pressão de perda do detrusor e a capacidade vesical máxima antes e depois do tratamento, além de registros no diário miccional em quatro graus, sendo 0 para completamente seco, 1 para molhado uma vez por dia, geralmente durante a noite, 2 para molhado em menos $50 \%$ do tempo acordado e 3 para molhado por mais de $50 \%$ do tempo. O número de trocas diárias de fralda também foi registrado no diário miccional. Os autores descreveram que dois dos doze pacientes $(16,6 \%)$ tornaram-se completamente secos, três crianças (25\%) permaneceram inalteradas, lembrando que destes, dois eram completamente incontinentes antes do tratamento, e os demais mantiveram ou diminuíram a pontuação diária no diário miccional, de grau 3 para grau 1. Para os autores o tratamento com FES diminuiu o número de perdas diurnas e noturnas, reduzindo a troca de fraldas em $75 \%$ dos casos, além de ser uma técnica bem tolerada pelas crianças.

Mais recentemente Azevedo et al. ${ }^{10}$ avaliou retrospectivamente 15 anos de tratamento multidisciplinar para 192 crianças e adolescentes (64\% meninas) com DTUI, e idades variando entre 0,1 a 16,8 anos. Um dos tratamentos foi a uroterapia, que consistia de treinamento comportamental, micção programada, hidratação oral, dieta laxativa, biofeedback e eletroestimulação sacral. Outras formas terapêuticas avaliadas foram o cateterismo vesical intermitente limpo, terapia anticolinérgica, enema retal, tratamento de infecção do trato urinário e, em casos refratários, procedimentos cirúrgicos.

Os pacientes foram avaliados na admissão e ao final do tratamento por meio de exames de imagem, particularmente a ultrassonografia renal e dinâmica da micção, uretrocistografia miccional ou cistografia radioisotópica e estudo urodinâmico para pacientes com DTUI de etiologia neurológica, e em pacientes com incontinência urinária associada com infecção do trato urinário inferior; cintilografia renal estática e cintilografia renal dinâmica. Além destes foi realizada ainda a urocultura, que poderia ser repetida durante o tratamento. A partir especialmente das medianas e de distribuições percentuais, os autores concluíram que houve uma redução significativa da incontinência urinária diurna, da enurese noturna monossintomática, da incontinência fecal, da infecção urinária e, os autores destacaram, principalmente, que o biofeedback e a estimulação elétrica podem reduzir as perdas urinárias de pacientes com distúrbio funcional do trato urinário inferior.

No mesmo ano Campos et al. ${ }^{11}$ compararam 47 crianças de cinco a dez anos de idade com enurese monossintomática, divididas aleatoriamente em dois grupos, um submetido à terapia comportamental associada aos exercícios do assoalho pélvico, e outro à terapia comportamental associada à administração de cloreto de oxibutinina, $0,2 \mathrm{mg} / \mathrm{Kg}$ duas vezes ao dia por três meses.

A fisioterapia do assoalho pélvico foi realizada durante três meses, consistindo principalmente no reconhecimento da musculatura e de treino de contração e relaxamento, este realizado uma vez na semana no consultório da fisioterapia e duas vezes por semana em domicílio com o auxílio dos pais. Para a terapia comportamental, cujo objetivo foi reeducar os hábitos das crianças, os pais foram orientados sobre a ingestão de líquidos e a hora programada, restringindo-se a cafeína apenas na parte da manhã e da tarde, refrigerantes somente após o almoço, mas permitindo o consumo de suco em geral e água sem restrição.

A posição miccional também foi orientada, sendo para as meninas sentar no vaso sanitário com os pés apoiados sobre uma superfície ou no chão, calcinhas até os tornozelos e apoio de cotovelos sobre joelhos com flexão anterior de tronco, e para os meninos o mesmo, mas sem a necessidade de sentar no vaso. A avaliação, antes e após o tratamento, consistiu de exame de urina, urocultura e diário miccional do sol-e-chuva, este último preenchido durante todo o tratamento, contabilizando-se especialmente o número de noites secas. Pais e crianças foram orientados sobre a ingesta hídrica e o correto posicionamento miccional. Os autores descreveram que, ao final do tratamento, o treinamento da musculatura do assoalho 
pélvico associado à terapia comportamental foi superior à terapia comportamental aliada a oxibutinina na redução dos sintomas de incontinência para crianças que apresentavam enurese monosintomática.

\section{DISCUSSÃO}

O presente estudo objetivou descrever as técnicas utilizadas nacionalmente no tratamento das disfunções miccionais infantis, revisando para este fim, ensaios clínicos randomizados e não randomizados, restritos a crianças.

Eletroestimulação em crianças obrigatoriamente é feita transcutaneamente, apesar do estudo de Kajbafzadeh, et al. ${ }^{9}$ ter posicionado sobre o púbis e uretra, o mais encontrado na literatura é a estimulação parassacral12,13,14, em seguida a eletroestimulação do nervo tibial posterior ${ }^{15}$. Assim também varia a frequência utilizada entre 10 e $20 \mathrm{HZ}$ e largura de pulso de 100 até 1000 microssegundos, revelando que é que ainda não existe evidencia da eficácia do uso da eletroestimulação na DTUI na infância ${ }^{16}$.

Uroterapia é o termo utilizado para orientações comportamentais e miccionais referentes ingestão hídrica adequada, micção de horário e não inibição do desejo miccional ${ }^{16}$. Azevedo et al $^{10}$ mostra que este tratamento tem bons resultados e deve ser individualizado. Da mesma forma, vários estudos ${ }^{17-21}$ tem utilizado esta terapia, com resultados promissores.

O TMAP mostrado nesta revisão, com ou sem o auxílio do sistema de biofeedback, foi eficaz na diminuição dos sintomas de IU das crianças afetadas, particularmente quando aliado à terapia comportamental ${ }^{10,11}$. De fato, o TMAP tem sido bastante usado nas pesquisas, porém a maioria não o utiliza isoladamente, normalmente associado ao biofeedback e ouras terapias $20,22,23$.

\section{CONCLUSÃO}

Neste estudo foi possível observar a escassez de pesquisas publicadas em revistas nacionais relacionadas a fisioterapia no tratamento das disfunções urinárias infantis, o que dificultou o alcance do objetivo do estudo. Porém, existem estudos publicados em outras línguas mostrando eficácia da Fisioterapia.
Dentre as técnicas mais utilizadas estão TMAP, a uroterapia e a eletroestimulação, de forma isolada ou associadas como exercícios de propriocepção e de conscientização do assoalho pélvico com a terapia comportamental e treino miccional, tanto para tratamento da DTUI infantil de origem neurogênica ou não-neurogênica, apresentando resultados promissores e encorajando o uso dos mesmos na pratica clinica

\section{REFERÊNCIAS}

VASCONCELOS, M.M.A.; LIMA, E.M.; VAZ, G.B.; SILVA, T.H.S. Disfunção do trato urinário inferior - um diagnóstico comum na prática pediátrica. J. Bras. Nefrol. [online]. 2013, vol.35, n.1, pp. 57-64. ISSN 0101-2800

ROBINSON, J.L.; FINLAY, J.C.; LANG, M.E.; BORTOLUSSI, R. Canadian Paediatric Society, Infectious Diseases and Immunization Committee, Community Paediatrics Committee. Urinary tract infections in infants and children: Diagnosis and management. Peadiatr Child Health. 2014 Jun;19(6):315-25

NEVÉUS, T.; VON GONTARD, A.; HOEBEKE, P.; HJÄLMÅS, K.; BAUER, S.; BOWER, W.; JORGENSEN, T.M.; RITTIG, S.; WALLE, J.V.; YEUNG, C.K.; DJURHUUS, J.C. The standardization of terminology of lower urinary tract function in children and adolescents: report from the Standardisation Committee of the International Children's Continence Society. J Urol. 2006 Jul;176(1):314-24.

RODRIGUEZ, S.E. Las Disfunciones Miccionales en la Infancia. Rev. chil. pediatr. [online]. 2004, vol.75, n.6, pp. 512 519. ISSN 0370-4106.

VAZ, G.T.; VASCONCELOS, M.M.; OLIVEIRA, E.A.; FERREIRA, A.L.; MAGALHÃES, P.G.; SILVA, F.M. et al. Prevalence of lower urinary tract symptoms in school-age children. Pediatr Nephrol 2012;27:597-603.

GONZÁLEZ, M.Á.S.J.; FERNÁNDEZ, P. Incontinencia y trastornos miccionales: ¿qué podemos hacer? Revista Pediatría de Atención Primaria. Lugo, España, v. 11, n. 44, p.1-30. 11 out. 2009

BALLEK, N.K.; MCKENNA, PH. Lower urinary tract dysfunction in childhood. Urologic clinics of North America. V. 37 n. 2, p.215-228.

SOCIEDADE BRASILEIRA DE UROLOGIA. Enurese: Diagnóstico e tratamento. Projeto diretrizes: Associação Médica Brasileira e Conselho Federal de Medicina. Brasil, p. 1-12. 27 jun. 2006.

KAJBAFZADEH, A-M.; SHARIFI-RAD, L.; DIANAT. S. Efficacy of transcutaneous functional electrical stimulation on uri- 
nary incontinence in myelomeningocele: results of a pilot study. Int Braz J Urol Off J Braz Soc Urol. outubro de 2010;36(5):614-20.

AZEVEDO, R.V.M.; OLIVEIRA, E.A.; VASCONCELOS, M.M.A.; CASTRO, B.A.C.; PEREIRA, F.R.; DUARTE, N.F.V.; et al. Impact of an interdisciplinary approach in children and adolescents with lower urinary tract dysfunction (LUTD). J. Bras. Nefrol. 2014;36(4):451-459

CAMPOS, R.M.; GUGLIOTTA, A.; IKARI, O.; PERISSINOTO, M.C.; LÚCIO, A.C.; MIYAOKA, R.; et al . Estudo comparativo, prospectivo e randomizado entre uroterapia e tratamento farmacológico em crianças com incontinência urinária. Einstein (São Paulo) [Internet]. 2013 June [cited 2016 Apr 03] ; 11( 2 ): 203-208

DE PAEPE, H.; RENSON, C.; HOEBEKE, P.; RAES, A.; VAN LAECKE, E.; VANDE, WALLE, J. The role of pelvic-floor therapy in the treatment of lower urinary tract dysfunctions in children. Scand J Urol Nephrol. 2002;36(4):260-7.

FARHAT, W.; BAGLI, D.J.; CAPOLICCHIO, G.; et al. The dysfunctional voiding scoring system: quantitative standardization of dysfunctional voiding symptoms in children. $\mathrm{K}$ Urol 2000;164:1011.

BOWER, W.F.; YEUNG, C.K. A review of non-invasive electro neuromodulation as an intervention for non-neurogenic bladder dysfunction in children. Neurourol Urodyn. 2004;23(1):63-7

LORDÊLO, P.; SOARES, P.V.; MACIEL, I.; MACEDO, A. Jr.; BARROSO, U. Jr. Prospective study of transcutaneous parasacral electrical stimulation for overactive bladder in children: long-term results. J Urol. 2009 Dec;182(6):2900-4. doi: 10.1016/j.juro.2009.08.058. Epub 2009 Oct 28

BARROSO, U. Jr ${ }^{1}$. LORDÊLO, P.; LOPES, A.A.; ANDRADE, J.; MACEDO, A. Jr.; ORTIZ, V. Nonpharmacological treatment of lower urinary tract dysfunction using biofeedback and transcutaneous electrical stimulation: a pilot study. BJU Int. 2006 Jul;98(1):166-71.

QUINTILIANO, F.; ${ }^{1}$ VEIGA, M.L ${ }^{1 . ;}$, MORAES, $M^{1},{ }^{\prime}$ CUNHA, $C^{1}$. DE OLIVEIRA, LF ${ }^{1}$. LORDELO, ${ }^{1}$. BASTOS NETTO, J. $M^{\prime \prime}$. BARROSO JÚNIOR, U. Transcutaneous parasacral electrical stimulation vs oxybutynin for the treatment of overactive bladder in children: a randomized clinical trial.UJ Urol. 2015 May;193(5 Suppl):1749-53. doi: 10.1016/j.juro.2014.12.001. Epub 2015 Mar 24.

CAPITANUCCI, M.L.; ${ }^{1}$ CAMANNI, D.; DEMELAS. F.; MOSIELLO, G.; ZACCARA, A.; DE GENNARO, M. Long-term efficacy of percutaneous tibial nerve stimulation for different types of lower urinary tract dysfunction in children. J Urol. 2009 Oct;182(4Suppl):2056-61.doi:10.1016/j.juro.2009.03.007. Epub 2009 Aug 20.
CORREIA, D.M.; SANTO, J.M.; FERREIRA, R.S.; BARROSO JUNIOR, U.; LORDÊLO, P.G. Estimulação elétrica neural para hiperatividade vesical: uma revisão sistemática. Revista Pesquisa em Fisioterapia. 2013 Dez;3(2):142-151

LADI, Seyedian S.S.; SHARIFI-RAD, L.; EBADI, M.; KAJBAFZADEH, A.M. Combined functional pelvic floor muscle exercises with Swiss ball and urotherapy for management of dysfunctional voiding in children: a randomized clinical trial. Eur J Pediatr. 2014 Oct;173(10):1347-53. doi: 10.1007/ s00431-014-2336-0. Epub 2014 May 21.

ZIVKOVIC, V.; LAZOVIC, M.; VLAJKOVIC, M.; SLAVKOVIC, A.; DIMITRIJEVIC, L.; STANKOVIC, I.; VACIC, N. Diaphragmatic breathing exercises and pelvic floor retraining in children with dysfunctional voiding. Eur J Phys Rehabil Med. 2012 Sep;48(3):413-21. Epub 2012 Jun 5.

KAJBAFZADEH, A.M.; SHARIFI-RAD, L.; GHAHESTANI, S.M.; AHMADI, H.; KAJBAFZADEH, M.; MAHBOUBI, AH. Animated biofeedback: an ideal treatment for children with dysfunctional elimination syndrome. J Urol. 2011 Dec; 186(6):237984. doi: 10.1016/j.juro.2011.07.118. Epub 2011 Oct 22.

BAEL, A.; LAX, H.; DE JONG, T.P.; HOEBEKE, P.; NIJMAN RJ, SIXT, R.; VERHULST, J.; HIRCHE, H.; VAN GOOL, J.D. European Bladder Dysfunction Study (European Union BMH1-CT94-1006). The relevance of urodynamic studies for Urge syndrome and dysfunctional voiding: a multicenter controlled trial in children. J Urol. 2008 Oct;180(4):1486-93; discussion 1494-5. doi: 10.1016/j.juro.2008.06.054. Epub 2008 Aug 16.

VESNA, Z.D.; MILICA, L.; STANKOVIĆ, I.; MARINA, V.; ANDJELKA, S. The evaluation of combined standard urotherapy, abdominal and pelvic floor retraining in children with dysfunctional voiding. J Pediatr Urol. 2011 Jun;7(3):336-41. doi: 10.1016/j.jpurol.2011.02.028. Epub 2011 Apr 27.

LADI-SEYEDIAN, S.; KAJBAFZADEH, A.M.; SHARIFI-RAD, L.; SHADGAN. B.; FAN, E. Management of non-neuropathic underactive bladder in children with voiding dysfunction by animated biofeedback: a randomized clinical trial. Urology. 2015 Jan;85(1):205-10. doi: 10.1016/j.urology.2014.09.025. Epub 2014 Nov 11

VASCONCELOS, M.; LIMA, E.; CAIAFA, L.; NORONHA, A.; CANGUSSU, R.; GOMES, S.; FREIRE, R.; FILGUEIRAS, M.T.; ARAÚJO, J.; MAGNUS, G.; CUNHA, C.; COLOZIMO, E. Voiding dysfunction in children. Pelvic-floor exercises or biofeedback therapy: a randomized study. Pediatr Nephrol. 2006 Dec;21(12):1858-64. Epub 2006 Sep 12. 